\title{
Properties of the Taylor Series Expansion Coefficients of the Jacobian Elliptic Functions
}

\author{
By Alois Schett
}

\begin{abstract}
Properties of the Taylor series expansion coefficients of the Jacobian elliptic functions and tables for the first fifteen leading terms are given. Relations of these coefficients with the randomization distributions are shown.
\end{abstract}

Little is known about the Taylor series expansion coefficients of the Jacobian elliptic functions $\operatorname{sn}(u, k), \operatorname{cn}(u, k)$ and $\operatorname{dn}(u, k)$. No recurrence formula exists for these coefficients. Only four to five leading terms of the series are given in literature ([1], [2]).

We present in this paper properties of these coefficients, show relations between them and randomization distributions [3, p. 51], and give tables for the first fifteen leading terms.

We consider the differential equations

$$
\begin{aligned}
& \frac{d}{d u} y_{1}(u)-C_{1} y_{2}(u) y_{3}(u)=0, \\
& \frac{d}{d u} y_{2}(u)-C_{2} y_{1}(u) y_{3}(u)=0, \\
& \frac{d}{d u} y_{3}(u)-C_{3} y_{1}(u) y_{2}(u)=0 .
\end{aligned}
$$

Solution functions of (1) for $C_{1}=1, C_{2}=-1, C_{3}=-k^{2}$ are the Jacobian elliptic functions $y_{1}=\operatorname{sn}(u, k), y_{2}=\operatorname{cn}(u, k), y_{3}=\operatorname{dn}(u, k)([1],[2])$.

The formal Taylor series of the functions $y_{1}, y_{2}, y_{3}$ read

$$
\begin{aligned}
& y_{1}(u)=\sum_{n=0}^{\infty} \frac{\left(u-u_{0}\right)^{n}}{n !}\left[\sum a_{j_{1} j_{2} j_{3}} C_{1}^{j_{1}} C_{2}^{j_{2}} C_{3}^{j_{3}} y_{10}^{i_{1}} y_{20}^{i_{2}} y_{30}^{i_{3}}\right], \\
& y_{2}(u)=\sum_{n=0}^{\infty} \frac{\left(u-u_{0}\right)^{n}}{n !}\left[\sum b_{h_{1} h_{2} h_{3}} C_{1}^{h_{1}} C_{2}^{h_{2}} C_{3}^{h_{3}} y_{10}^{s_{1}} y_{20}^{s_{2}} y_{30}^{s_{3}}\right], \\
& y_{3}(u)=\sum_{n=0}^{\infty} \frac{\left(u-u_{0}\right)^{n}}{n !}\left[\sum c_{r_{1} r_{2} r_{3}} C_{1}^{r_{1}} C_{2}^{r_{2}} C_{3}^{\left.r_{3} y_{10}^{q_{1}} y_{20}^{q_{2}} y_{30}^{q_{3}}\right]} .\right.
\end{aligned}
$$

The summation over the indices $j_{1}, j_{2}, j_{3} ; h_{1}, h_{2}, h_{3} ; r_{1}, r_{2}, r_{3}$ and their relation to the exponents of $y_{10}, y_{20}, y_{30}$ are specified in Theorem I.

$$
y_{m 0}=y_{m}\left(u_{0}\right) \quad(m=1,2,3) .
$$

For $u_{0}=0, y_{10}=0, y_{20}=y_{30}=1$, this series is convergent in the region $|u|<K^{\prime}$ [2], where

Received October 14, 1974; revised March 3, 1975 and May 27, 1975.

AMS (MOS) subject classifications (1970). Primary 33A25, 65A05; Secondary 05A99. 


$$
K^{\prime}=K\left(k^{\prime}\right)=\int_{0}^{\pi / 2} \frac{d \theta}{\sqrt{ }\left(1-k^{\prime 2} \sin ^{2} \theta\right)}, \quad k^{\prime}=\sqrt{1-k^{2}} .
$$

For the explicit series of (2), the elements $a_{j_{1} j_{2} j_{3}}, b_{h_{1} h_{2} h_{3}}, c_{r_{1} r_{2} r_{3}}$ have to be determined and summation over the indices has to be specified.

THEOREM I. $a_{j_{1} j_{2} j_{3}} \neq 0$ only for

$$
\begin{array}{ll}
j_{1}=1,2, \ldots, J_{1} ; & J_{1}= \begin{cases}n / 2 & \text { for } n \text { even }, \\
(n+1) / 2 & \text { for } n \text { odd },\end{cases} \\
j_{2}=0,1, \ldots, J_{2} ; \\
j_{3}=0,1, \ldots, J_{3} ;
\end{array} \quad J_{2}, J_{3}= \begin{cases}n / 2 & \text { for } n \text { even, } \\
(n-1) / 2 & \text { for } n \text { odd },\end{cases}
$$

and $j_{1}, j_{2}, j_{3}$ satisfying the relation $j_{1}+j_{2}+j_{3}=n . i_{1}, i_{2}, i_{3}$ is obtained from the relations $i_{1}=n+1-2 j_{1}, i_{2}=n-2 j_{2}, i_{3}=n-2 j_{3} . b_{h_{1} h_{2} h_{3}} \neq 0$ only for $h_{1}=j_{3}$, $h_{2}=j_{1}, h_{3}=j_{2}$.

The exponents $s_{1}, s_{2}, s_{3}$ are related to $h_{1}, h_{2}, h_{3}$ as follows: $s_{1}=n-2 h_{1}, s_{2}=$ $n+1-2 h_{2}, s_{3}=n-2 h_{3} . c_{r_{1} r_{2} r_{3}} \neq 0$ only for $r_{1}=j_{2}, r_{2}=j_{3}, r_{3}=j_{1}$. The exponents $q_{1}, q_{2}, q_{3}$ are defined by the relations $q_{1}=n-2 r_{1}, q_{2}=n-2 r_{2}, q_{3}=$ $n+1-2 r_{3}$.

For $u_{0}=0$, i.e.,

$$
\begin{aligned}
y_{1}\left(u_{0}=0\right)= & \operatorname{sn}(0, k)=0, \quad y_{2}\left(u_{0}=0\right)=\operatorname{cn}(0, k)=1, \\
& y_{3}\left(u_{0}=0\right)=\operatorname{dn}(0, k)=1,
\end{aligned}
$$

we obtain

$$
\begin{aligned}
& a_{j_{1} j_{2} j_{3}} \neq 0 \text { only for } j_{1}=(n+1) / 2 ; j_{2}=0,1, \ldots,(n-1) / 2 ; n \text { odd, } \\
& b_{h_{1} h_{2} h_{3}} \neq 0 \text { only for } h_{3}=n / 2 ; h_{1}=0,1, \ldots, n / 2-1 ; n \text { even, } \\
& c_{r_{1} r_{2} r_{3}} \neq 0 \text { only for } r_{2}=n / 2 ; r_{3}=1,2, \ldots, n / 2 ; n \text { even. }
\end{aligned}
$$

In the following table the elements $a_{j_{1} j_{2} j_{3}}, b_{h_{1} h_{2} h_{3}}, c_{r_{1} r_{2} r_{3}}$ and the exponents $i_{1}, i_{2}, i_{3} ; s_{1}, s_{2}, s_{3} ; q_{1}, q_{2}, q_{3}$ are given as an illustration for $n=3$ and $n=4$.

TABLE I

$$
\begin{array}{rrrrrrrrrrrrrrrrrrrrrrrrrrr}
n=3 & a_{j_{1} j_{2} j_{3}} & j_{1} & j_{2} & j_{3} & i_{1} & i_{2} & i_{3} & b_{h_{1} h_{2} h_{3}} & h_{1} & h_{2} & h_{3} & s_{1} & s_{2} & s_{3} & c_{r_{1} r_{2} r_{3}} & r_{1} & r_{2} & r_{3} & q_{1} & q_{2} & q_{3} \\
& 1 & 2 & 1 & 0 & 0 & 1 & 3 & 4 & 1 & 1 & 1 & 1 & 2 & 1 & 4 & 1 & 1 & 1 & 1 & 1 & 2 \\
& 1 & 2 & 0 & 1 & 0 & 3 & 1 & 1 & 1 & 2 & 0 & 1 & 0 & 3 & 1 & 1 & 0 & 2 & 1 & 3 & 0 \\
& 4 & 1 & 1 & 1 & 2 & 1 & 1 & 1 & 0 & 2 & 1 & 3 & 0 & 1 & 1 & 0 & 1 & 2 & 3 & 1 & 0 \\
n=4 & 14 & 2 & 1 & 1 & 1 & 2 & 2 & 4 & 2 & 1 & 1 & 0 & 3 & 2 & 4 & 2 & 1 & 1 & 0 & 2 & 3 \\
& 1 & 2 & 2 & 0 & 1 & 0 & 4 & 1 & 2 & 2 & 0 & 0 & 1 & 4 & 1 & 2 & 0 & 2 & 0 & 4 & 1 \\
& 4 & 1 & 2 & 1 & 3 & 0 & 2 & 14 & 1 & 2 & 1 & 2 & 1 & 2 & 14 & 1 & 1 & 2 & 2 & 2 & 1 \\
& 1 & 2 & 0 & 2 & 1 & 4 & 0 & 4 & 1 & 1 & 2 & 2 & 3 & 0 & 4 & 1 & 2 & 1 & 2 & 0 & 3 \\
4 & 1 & 1 & 2 & 3 & 2 & 0 & 1 & 0 & 2 & 2 & 4 & 1 & 0 & 1 & 0 & 2 & 2 & 4 & 0 & 1
\end{array}
$$


The following identities and symmetries are valid:

THEOREM II.

$$
\begin{aligned}
& a_{j_{1} j_{2} j_{3}}=b_{j_{3} j_{1} j_{2}}=c_{j_{2} j_{3} j_{1}}, \\
& a_{j_{1} j_{2} j_{3}}=a_{j_{1} j_{3} j_{2}}
\end{aligned}
$$

and therefore,

$$
\begin{aligned}
& b_{j_{3} j_{1} j_{2}}=b_{j_{2} j_{1} j_{3}}, \\
& c_{j_{2} j_{3} j_{1}}=c_{j_{3} j_{2} j_{1}} .
\end{aligned}
$$

Theorems III/1, III/2 and III/3 show relations between the randomization distribution and the elements $a_{j_{1} j_{2} j_{3}}, b_{h_{1} h_{2} h_{3}}$ and $c_{r_{1} r_{2} r_{3}}$.

THEOREM III/1. The sum of all elements $a_{j_{1} j_{2} j_{3}}$ for a given $n$ is equal to $n$ !.

Example: $n=4$.

$$
a_{112}+a_{121}+a_{202}+a_{211}+a_{220}=4+4+1+14+1=24=4 \text { ! }
$$

THEOREM III/2. For a given $n=2$, the following relation is valid:

$$
\sum_{j_{3}=0}^{J_{3}} a_{j_{1} j_{2} j_{3}} \geqslant R U_{j_{2}}, \quad j_{2}=0,1, \ldots, J_{2},
$$

where $R U_{j_{2}}$ is the number of permutations of n natural numbers with $j_{2}$ runs up. Tables of the numbers $R U_{j_{2}}$ are given in [3, p. 260, Table 7.2.2].

Example: $n=3$.

$$
a_{201}=1=R U_{0}, \quad a_{210}+a_{111}=1+4=5=R U_{1} .
$$

$\begin{array}{lll}\text { Permutations } & \begin{array}{l}\text { One run up } \\ \text { (underlined) }\end{array} & \begin{array}{l}\text { Zero run up } \\ \text { (underlined) }\end{array}\end{array}$

$\begin{array}{cccc} & 123 & \underline{123} & 123 \\ & 132 & \underline{132} & 132 \\ 231 & \underline{231} & 231 \\ & 213 & \underline{213} & 213 \\ 312 & \underline{312} & 312 \\ & 321 & 321 & \underline{321} \\ \text { Total } & 6=n ! & 5=R U_{1} & 1=R U_{0}\end{array}$

THEOREM III/3. For a given $n \geqslant 2$ the following relation is valid:

$$
\sum_{j_{2}=0}^{J_{2}} a_{j_{1} j_{2} j_{3}}=P_{j_{1}}, \quad j_{1}=1,2, \ldots, J_{1},
$$

where $P_{j_{1}}$ is the number of permutations of $n$ natural numbers with $j_{1}-1$ peaks. The 
numbers $P_{j_{1}}$ are tabulated in $[3, p .261$, Table 7.3].

Example: $n=3$.

\begin{tabular}{|c|c|c|c|}
\hline & Permutations & $\begin{array}{l}\text { One peak } \\
\text { (underlined) }\end{array}$ & $\begin{array}{c}\text { Zero peak } \\
\text { (underlined) }\end{array}$ \\
\hline & 123 & 123 & 123 \\
\hline & 132 & 132 & 132 \\
\hline & 231 & 231 & 231 \\
\hline & 213 & 213 & $\underline{213}$ \\
\hline & 312 & 312 & $\underline{312}$ \\
\hline & 321 & 321 & $\underline{321}$ \\
\hline Total & $6=n !$ & $2=P_{2}$ & $4=P_{1}$ \\
\hline
\end{tabular}

Similar results can be obtained for $b_{h_{1} h_{2} h_{3}}$ and $c_{r_{1} r_{2} r_{3}}$ using Theorem II.

These theorems can be proved by mathematical induction. Theorem III/ 1 follows from Theorem III/ 2 or Theorem III/ 3 since the number of permuations of $n$ natural numbers is equal to $n !$.

Table II, in the microfiche section attached to this issue, lists the elements $a_{j_{1} j_{2} j_{3}}$ for $n=0,1,2, \ldots, 15$.

Putting $u_{0}=0$ the explicit terms of the series for $\operatorname{sn}(u, k), \operatorname{cn}(u, k)$ and $\operatorname{dn}(u, k)$ read (Theorem I, Theorem II and Table II):

$$
\begin{aligned}
\operatorname{sn}(u, k)= & u-\left(1+k^{2}\right) \frac{u^{3}}{3 !}+\left(1+14 k^{2}+k^{4}\right) \frac{u^{5}}{5 !}-\left(1+135 k^{2}+135 k^{4}+k^{6}\right) \frac{u^{7}}{7 !} \\
& +\left(1+1228 k^{2}+5478 k^{4}+1228 k^{6}+k^{8}\right) \frac{u^{9}}{9 !} \\
& -\left(1+11069 k^{2}+165826 k^{4}+165826 k^{6}+11069 k^{8}+k^{10}\right) \frac{u^{11}}{11 !} \\
& +\left(1+99642 k^{2}+4494351 k^{4}+13180268 k^{6}+4494351 k^{8}\right. \\
& -\left(1+896803 k^{2}+116294673 k^{4}+834687179 k^{6}+834687179 k^{8}\right. \\
& +\sum_{n_{0}=1 ;\left(n_{0} \text { odd }\right)}^{\infty}(-1)^{\left(n_{0}-1\right) / 2}\left(\sum_{j_{2}=0}^{\left(n_{0}-1\right) / 2} a_{j_{1} j_{2} j_{3}} k^{2 j_{2}}\right) \frac{u^{n_{0}}}{n_{0} !}, j_{1}=\frac{n_{0}+1}{2} \\
= &
\end{aligned}
$$

(terms for $n_{0} \leqslant 7$ are given in [2]); 


$$
\begin{aligned}
\operatorname{cn}(u, k)= & 1-\frac{u^{2}}{2 !}+\left(1+4 k^{2}\right) \frac{u^{4}}{4 !}-\left(1+44 k^{2}+16 k^{4}\right) \frac{u^{6}}{6 !} \\
& +\left(1+408 k^{2}+912 k^{4}+64 k^{6}\right) \frac{u^{8}}{8 !} \\
& -\left(1+3688 k^{2}+30768 k^{4}+15808 k^{6}+256 k^{8}\right) \frac{u^{10}}{10 !} \\
& +\left(1+33212 k^{2}+870640 k^{4}+1538560 k^{6}+259328 k^{8}+1024 k^{10}\right) \frac{u^{12}}{12 !} \\
& -\left(1+298932 k^{2}+22945056 k^{4}+106923008 k^{6}+65008896 k^{8}\right. \\
= & 1+\sum_{n_{e}=2 ;\left(n_{e} \text { even }\right)}^{\infty}(-1)^{n_{e} / 2}\left(\sum_{h_{1}=0}^{n_{e} / 2-1} b_{\left.h_{1} h_{2} h_{3} k^{2 h_{1}}\right)}\right) \frac{u^{n_{e}}}{n_{e} !}, \quad h_{3}=n_{e} / 2
\end{aligned}
$$

(terms for $n_{e} \leqslant 8$ are given in [2]);

$$
\begin{aligned}
\operatorname{dn}(u, k)= & 1-k^{2} \frac{u^{2}}{2 !}+\left(4+k^{2}\right) k^{2} \frac{u^{4}}{4 !}-\left(16+44 k^{2}+k^{4}\right) k^{2} \frac{u^{6}}{6 !} \\
& +\left(64+912 k^{2}+408 k^{4}+k^{6}\right) k^{2} \frac{u^{8}}{8 !} \\
& -\left(256+15808 k^{2}+30768 k^{4}+3688 k^{6}+k^{8}\right) \frac{u^{10} k^{2}}{10 !} \\
& +\left(1024+259328 k^{2}+1538560 k^{4}+870640 k^{6}+33212 k^{8}+k^{10}\right) \frac{u^{12} k^{2}}{12 !} \\
& -\left(4096+4180992 k^{2}+65008896 k^{4}+106923008 k^{6}\right. \\
= & 1+\sum_{n_{e}=2 ;\left(n_{e} \text { even }\right)}^{\infty}(-1)^{n} e^{/ 2}\left(\sum_{r_{3}=1}^{n_{e} / 2} c_{r_{1} r_{2} r_{3}} k^{2\left(r_{3}-1\right)}\right) \frac{u^{n_{e}} k^{2}}{n_{e} !}, \quad r_{2}=n_{e} / 2
\end{aligned}
$$

(terms for $n_{e} \leqslant 8$ are given in [2]).

CEN

Saclay, France

1. M. ABRAMOWITZ \& I. A. STEGUN (Editors), Handbook of Mathematical Functions, With Formulas, Graphs and Mathematical Tables, 5th printing with corrections, Nat. Bur. Standards Appl. Math. Ser., vol. 55, Superintendent of Documents, U. S. Government Printing Office, Washington, D. C., 1966, p. 575. MR $34 \# 8607$.

2. P. F. BYRD \& M. D. FRIEDMAN, Handbook of Elliptic Integrals for Engineers and Scientists, 2nd rev. ed., Die Grundlehren der math. Wissenschaften, Band 67, Springer-Verlag, New York, 1971, p. $303 . \quad$ MR 43 \#3506.

3. F. N. DAVID, M. G. KENDALL \& D. E. BARTON, Symmetric Function and Allied Tables, Cambridge Univ. Press, Cambridge, 1966. MR 34 \#2099. 